BULLETIN OF THE

AMERICAN MATHEMATICAL SOCIETY

Volume 80, Number 3, May 1974

\title{
COMPLETE CONVEX HYPERSURFACES OF A HILBERT SPACE
}

\author{
BY RUBENS LEÃO DE ANDRADE
}

Communicated by S. S. Chern, February 18, 1973

A complete convex hypersurface of a (separable) Hilbert space $H$ is a codimension one $C^{\infty}$ submanifold of $H$, which is complete as a metric subspace of $H$ and such that $M=\partial K$, where $K$ is a (closed) convex set with nonvoid interior. For each $p \in M$ let $v(p)$ be the unit normal vector which points to the interior of $K$. In this way we define the Gauss map $\nu: M \rightarrow \Sigma$ from $M$ into the unit sphere $\Sigma$ of $H$. This is a $C^{\infty}$ map and its derivative at each point $p \in M$ is selfadjoint. We say that $M$ bounds $a$ half-line if there exists a half-line $\{p+t v ; t \geqq 0\}$ contained in the interior of $K$. In the finite dimensional case the condition that $M$ bounds a halfline is equivalent to that $M$ is unbounded. In the infinite dimensional case this is not true, as the following simple example shows. Let $A$ be a compact positive definite selfadjoint operator in $H$ and set $M=\{x \in H$; $\langle A(x), x\rangle=1\}$. It is not difficult to prove that $M$ is an unbounded positively-curved convex hypersurface and that $M$ does not bound any halfline.

In this note we announce some properties of a complete convex hypersurface $M$ of a Hilbert space. Theorem A characterizes the three possible boundedness situations (bounded, unbounded and bounding a half-line, unbounded and bounding no half-line) in terms of the Gauss map of $M$. Theorem B gives a necessary and sufficient condition for $M$ to be a pseudograph (see definition below) over one of its tangent hyperplanes. Theorem $\mathrm{C}$ is the analogue of the Bonnet-Myers theorem for hypersurface of a Hilbert space. These results are part of my doctoral dissertation. I wish to thank my advisor Professor Manfredo do Carmo for suggesting these problems and for helpful conversations.

THEOREM A. Let $M$ be a complete convex hypersurface of a Hilbert space $H$. Then:

(1) $M$ is bounded iff the Gauss map $v: M \rightarrow \Sigma$ is onto.

(2) $M$ is unbounded and bounds a half-line iff the image of the Gauss map is contained in a hemisphere.

(3) $M$ is unbounded and does not bound any half-line iff the image of the Gauss map is dense and has void interior.

AMS (MOS) subject classifications (1970). Primary 58B20; Secondary 53C40. 
Before stating Theorem B, we need to rephrase slightly the definition of pseudograph given in [2]. A convex hypersurface $M$ is a pseudograph over the tangent hyperplane $F$ when:

(a) $M$ lies in one of the closed half-spaces determined by $F$,

(b) let $\pi: M \rightarrow F$ be the orthogonal projection and set $A=\pi(M)$. Then over the interior int $A, M$ is the graph of a $C^{\infty}$ function,

(c) for every $a \in A$-int $A, M \cap \pi^{-1}$ (a) is a closed half-line,

(d) for every hyperplane $L$ above $F, M \cap L$ is bounded.

In the case that $M$ is finite dimensional, the above reduces to the definition given in [2].

THEOREM B. Let $M$ be a complete convex hypersurface of a Hilbert space $H$. Then $M$ is unbounded and $\operatorname{int}(\nu(M)) \neq \varnothing$ iff $M$ is a pseudograph over one of its tangent hyperplanes $T M_{p} \neq M$.

Theorem C (The Bonnet-Myers Theorem for Hilbert HyperSURFACE). Let $M$ be a complete connected hypersurface of a Hilbert space $H$. If the sectional curvatures of $M$ are all bounded away from zero (i.e. there exists a $\delta>0$ such that for every $p \in M$ and every two-plane section $\sigma \subset T M_{p}$ one has $K(\sigma) \geqq \delta)$ then $M$ is bounded, the diameter $\rho$ of $M$ satisfies $\rho \leqq \pi / \sqrt{ } \delta$ and the Gauss map is a diffeomorphism.

REMARK 1. Theorem B should be compared with a theorem of $\mathrm{H}$. H. Wu [2]. It should be remarked that Wu also proved that if $M$ is a complete convex hypersurface of $R^{n}$, then $\operatorname{int}(v(M))=\operatorname{int} \overline{(v(M))}$.

Theorem A shows that in the infinite dimensional case, this equality does not hold and we may have the extremal case in which $\operatorname{int}(v(M))=\varnothing$ and $\operatorname{int} \overline{(v(M))}=\Sigma$. This explains why we need the condition $\operatorname{int}(v(M)) \neq \varnothing$ in Theorem B, in contrast with Wu's theorem, where no such condition is required.

REMARK 2. The hypothesis of Theorem B is implied by the following condition on the sectional curvature of $M$ (see [1]): The sectional curvatures of $M$ are everywhere nonnegative and at some point $p \in M$ are all bounded away from zero. Thus in the finite dimensional case, Theorem B reduces to Wu's theorem.

\section{REFERENCES}

1. Leo Jonker, Hypersurfaces of nonnegative curvatures in Hilbert space, Trans. Amer. Math. Soc. 169 (1972), 461-474.

2. $\mathrm{H}$. Wu, $A$ structure theorem for complete noncompact hypersurfaces of nonnegative curvature, Bull. Amer. Math. Soc. 77 (1971), 1070-1071. MR 44 \#2183.

Instituto de Matemática Pura e Aplicada, Rio de Janeiro, Brasil 